\title{
Remisyonda olan ankilozan spondilit hastasında tedavi kararı
}

\author{
Treatment decision in patient with ankylosing spondylitis in remission \\ Hüseyin Turgut Elbek Özer@ \\ Çukurova Üniversitesi Tıp Fakültesi, İç Hastalıkları Anabilim Dalı, Romatoloji-İmmünoloji Bilim Dalı, \\ Adana, Türkiye
}

\section{Öz}

Ankilozan spondilitte non-steroid anti-inflamatuvar ilaçlarda (NSAii) \%9-35'e varan oranlarda anti-TNF ajanlarında \%16-62 oranlarında remisyon sağlandığı görülmüştür. Biyolojik ilaç kesimini takiben bir yıl içerisinde hastalarda \%47-76 oranlarında relaps görülmüştür. Remisyon sonrası doz azaltılması, tam doz tedavi devamı ile karşılaştırıldığında benzer oranlarda remisyon sürdürülmüştür (\%53,5'e karşı \%61,4). Ankilozan spondilitte NSAil'ın kullanımının radyolojik progresyonu gerilettiği ve azalmış mortalite ile ilişkili olduğu gösterilmiştir. NSAili'ın gerektiği durumlarda kullanılması önerilmektedir. Egzersiz programlarına remisyon sonrasında da devam edilmelidir.

Anahtar Sözcükler: Ankilozan spondilit, remisyon, non-steroid anti-inflamatuvar ilaçlar, anti-TNF, biyolojik ilaçlar.

\begin{abstract}
Non-steroidal anti-inflammatory drugs (NSAIDs) may attain 9 to 35\% remission rates in patients with ankylosing spondylitis, this rate may rise to 16 to $62 \%$ in anti-TNF users. Following withdrawal of biologics there is 47 to $76 \%$ relapse rate in one year. Dose reduction or standard dosing of anti-TNF therapies yields comparable rates of remission maintenance in one year (53.5\% vs. $61.4 \%)$. NSAIDs have shown to retard radiologic progression and decrease mortality in AS patients. NSAIDs should be used on demand in stable patients. Physical therapy is also recommended after the remission.
\end{abstract}

Keywords: Ankylosing spondylitis, remission, non-steroidal anti-inflammatory drug, anti-TNF, biologic agents.

Ankilozan spondilit (AS)'te remisyon, ASAS (Assesment of Spondyloarthritis International Society) parsiyel remisyon kriterlerini karşılıma veya ASDAS (ankilozan spondilit hastalık aktivasyon skoru) skorunda 1,3'ten küçük değerler olarak tanımlanmaktadır (1).

\section{NSAil' larda değişen oranlarda remisyon elde edilebilmektedir;}

Remisyon etorikoksibde altıncı haftada \%9,117,6 oranlarında görülmüştür (2). INFAST çalışması naproksenle 28 haftada ASAS parsiyel remisyon kriterlerine göre \%35,3'e varan oranlarında remisyon sağlanabileceğini göstermiştir (3). Biyolojiklerde ise (NSAil ile beraber) \%17-61,9 düzeyinde remisyon oranları bildirilmiştir (Tablo-1). Yine INFAST çalışmasında ASDAS-CRP $<1,3$ oranları plasebo + naproksen grubunda \%19,6 iken bu oran infliksimab + naproksen grubunda \%51,4'e çıkmıştır (3).

\section{Biyolojik ajanlarda durum nedir?}

Elli üç ADA, 78 ETA, 154 INF kullanan AS hastalarında parsiyel remisyon oranları 24 . haftada $\% 62$ iken, yan etkiler nedeniyle ikinci anti-TNF'ye geçilen hastalarda bu oran $\% 50$, etkisizlik nedeniyle geçilenlerde ise \%40'a düşmektedir (13).

Aksiyel spondiloartriti olan 46 hastanın bir yıllık adalimumab tedavisi sonrasında $\% 57,9$ oranında ASAS kısmi remisyon sağlanmıştır. Adalimumub tedavisi kesilmesinden sonra birinci yılda 24 hastanın 19'unda (\%79) relaps gözlenmiş, tedavi sonrasında 48 haftada \%47 oranında remisyon sağlanmıştır (14). 
Tablo-1. Ankilozan spondilitte Anti-TNF ajanların remisyon oranları.

\begin{tabular}{|c|c|c|c|c|c|c|}
\hline İlaç & Grup & $\mathbf{N}$ & $\begin{array}{l}\text { Remisyon } \\
\text { Tanımı }\end{array}$ & $\begin{array}{l}\text { Süre } \\
\text { (hafta) }\end{array}$ & $\begin{array}{l}\text { Remisyon } \\
\text { oranı (\%) }\end{array}$ & Çalışma \\
\hline İnfliksimab & AS & 201 & ASAS & 24 & 22,4 & ASSERT (4) \\
\hline Etanersept & AS & 138 & ASAS & 24 & 17 & Davis ve ark. (5) \\
\hline Adalimumab & AS & 208 & ASAS & 24 & 22,1 & ATLAS (6) \\
\hline Golimumab & AS & 138 & ASAS & 24 & 23,1 & GO-RAISE (7) \\
\hline \multirow[t]{2}{*}{$\begin{array}{l}\text { Sertolizumab } \\
\text { pegol }\end{array}$} & Aks SpA & 111 & ASAS & 24 & 23,4 & RAPID (8) \\
\hline & & & ASDAS & & 29,7 & \\
\hline İnfliksimab & Aks SpA & 20 & ASAS & 16 & 55,6 & Barkham ve ark (9) \\
\hline \multirow[t]{2}{*}{ İnfliksimab } & Aks SpA & 106 & ASAS & 28 & 61,9 & INFAST (3) \\
\hline & & & ASDAS & & 51,4 & \\
\hline \multirow[t]{2}{*}{ Etanersept } & Aks Spa & 40 & ASAS & 48 & 50 & ESTHER (10) \\
\hline & & & ASDAS & & 33 & \\
\hline \multirow[t]{2}{*}{ Adalimumab } & Nr-Aks SpA & 91 & ASAS & 12 & 16 & ABILITY-1 (11) \\
\hline & & & ASDAS & & 24 & \\
\hline Etanersept & Nr-Aks SpA & 106 & ASDAS & 12 & 40 & B1801031 (12) \\
\hline
\end{tabular}

AS: ankilozan spondilit, Aks SpA: aksiyel spondiloartrit, Nr-Aks SpA: radyolojik olmayan akiyel spondiloartrit, ASAS: Assesment of Spondyloarthritis International Society, ASDAS: ankilozan spondilit hastalık aktivasyon skoru.

Ankilozan spondilit hastalarında remisyondan sonra etanersept (ETA) dozu yarıya indirilirse ne olur sorusuna yanıt bulmak üzere gerçekleştirilen çalışmada; remisyona giren 22 hastaya iki haftada bir, 21 hastaya da her hafta $50 \mathrm{mg}$ ETA verildi. Grupların relaps oranları sırasıyla \%13,6 ve $\% 9,5$, relaps süresi $8 \pm 3,2$ ay ve $10 \pm 1,1$ ay bulundu (15).

Randomize çift kör ABILITY-3 çalışmasında, remisyonun sürdürülmesinde adalimumab (ADA) tedavisinin devamı veya çekilmesi sorgulandı. ADA'ya devam eden 144 hasta ve kesilen 140 hasta protokolü tamamladı. Devam grubunda 68 . haftada hastaların \%70'inde alevlenme gözlenmezken, bu oran plasebo grubunda \%47 bulundu. On iki haftalık kurtarma tedavisinde benzer oranlarda (\% 56 ve 57 ) remisyon sağlandı (16).

REMINEA çalışması infliksimab (INF) çekilince ne oluyor, sorusuna yanıt aramıştır. Yüz yedi hastalık AS kohortunda, sürekli remisyonda olan 36 hastanın tedavisinin kesilmesinin birinci yılında relaps gelişmiştir. Tekrar INF verilmesiyle 11 hastada remisyon sağlanmış, yedi hastada iyi klinik yanıt alınmıştır (17).
AS'de, referans infilksimab ile remisyon sağlandıktan sonra, biyobenzer ile remisyon sürdürülüyor mu? Kaltsonoudis ve ark.'larının 18 haftalık açık etiketli çalışmasında; 40'ar hastadan oluşan her iki kolda ortalama ASDAS skorlarının benzer olduğu gözlenmiştir (1,0'a karşı 1,1) (18).

TNF inhibitörlerinde doz azaltılmasının standart dozdan farkı var mı? REDES-TNF çalışmasında, ADA dozu üç haftada bir, ETA dozu 10 günde bir, golimumab dozu altı haftada bir ve INF dozu (3 $\mathrm{mg} / \mathrm{kg}$ ) sekiz haftada bir verilen 60 hasta, standart doz verilen 60 hastayla karşılaştırıldı. Bir yıl sonunda ASDAS-CRP remisyon oranları standart doz grubunda $\% 61,4$ diğer grupta ise \%53,5 ( $\mathrm{P}=0.389)$ olarak bildirildi (19).

\section{Spondiloartritlerde relaps için risk faktörleri nelerdir?}

CRESPA çalışmasında; ASAS kriterlerine göre periferik spondiloartrit (SpA) tanılı 60 hastanın sonuçlarına bakıldığında; golimumab ile indüksiyon tedavisi sonrasında ilaç kesildiğinde hastaların \%47'sinde ortalama 32 hafta sonra relaps geliştiği görüldü. Şiş eklem sayısının beşten fazla olması ve psoriazisin varlığı relaps 
için risk oluşturdu. HLA-B27 negatif hastaların remisyonda kalma şansları daha yüksek bulundu (20).

ABILITY-3 çalışmasında; radyolojik olmayan aksiyel $\mathrm{SpA}$ tanısı olan ve açık etiketli ADA kullanan hastalarda remisyon belirleyicilerine bakıldı. HLA-B27 pozitifliği, 45 yaştan genç olma, erkek cinsiyet 12 haftada remisyon için belirleyici bulundu (21).

\section{Remisyon sonrası NSAil'lara devam edelim mi?}

Selekoksible yapılan bir çalışmada sürekli NSAil kullanımın, ihtiyaç halinde kullanıma göre iki yıllık radyolojik progresyonu daha fazla azalttığı gösterilmiştir (22). Yine NSAil ile yapılan başka bir çalışmada normal CRP düzeyli AS hastalarında sürekli NSAil kullanan grupta aralıklı kullanana göre daha fazla radyolojik ilerleme; yüksek CRP düzeyli grupta ise sürekli NSAil kullananlarda daha az radyolojik ilerleme gösterilmiştir (23). Çok merkezli ANRADA çalışmasında sürekli diklofenak verilen hastalarda intiyaç halinde verilenlere göre daha fazla progresyon görülmüştür (24).
AS'te mortalitenin hastalık aktivitesi ile ilişkili olduğu gösterilmiştir. Artmış mortalite ile ilişkili bağımsız faktörler arasında yüksek CRP, NSAil'ların seyrek kullanımı, tanı gecikmesi, iş gücü kaybı bildirilmiştir (25). Toplum bazlı başka bir çalışmada da AS hastalarında artmış kadiovasküler ve serebrovasküler mortalite bulunmuş olup, risk faktörleri arasında erkek cinsiyet, düşük gelir, kronik böbrek hastalığı, periferik vasküler hastalık, 65 yaş ve üstünde ise NSAil ve statinlerin kullanılmaması gösterilmiştir (26). Bu veriler hastalık progresyonunun azaltılmasına katkısı olan NSAil kullanımın uygun hastalarda kaçınılmaması gerektiğini düşündürtmektedir.

Stabil hastalarda, ilaç tedavisi dışında rehabilitasyona devam edilmelidir (27).

\section{Sonuç}

AS'te remisyon sağlandıktan sonra uygun hastalarda biyolojik tedavinin doz azaltılması veya periyod uzatılmasına gidilebilir. NSAil'ların gerekli durumlarda kullanımına devam edilmelidir.

\section{Kaynaklar}

1. Poddubnyy D, Gensler LS. Spontaneous, drug-induced, and drug-free remission in peripheral and axial spondyloarthritis. Best Pract Res Clin Rheumatol 2014 Oct; 28 (5): 807-18.

2. van der Heijde D, Baraf HS, Ramos-Remus C, et al. Evaluation of the efficacy of etoricoxib in ankylosing spondylitis: results of a fifty-two-week, randomized, controlled study. Arthritis Rheum 2005; 52: 1205-15.

3. Sieper $\mathrm{J}$, Lenaerts $\mathrm{J}$, Wollenhaupt $\mathrm{J}$, et al. Efficacy and safety of infliximab plus naproxen versus naproxen alone in patients with early, active axial spondyloarthritis: results from the double-blind, placebo-controlled INFAST study, Part 1. Ann Rheum Dis 2014; 73: 101-7.

4. van der Heijde D, Dijkmans B, Geusens $P$, et al. Efficacy and safety of infliximab in patients with ankylosing spondylitis: results of a randomized, placebo-controlled trial (ASSERT) Arthritis Rheum 2005;52:582-91.

5. Davis JC, Jr, Van Der Heijde D, Braun J, et al. Recombinant human tumor necrosis factor receptor (etanercept) for treating ankylosing spondylitis: a randomized, controlled trial. Arthritis Rheum 2003; 48: 3230-6.

6. van der Heijde D, Kivitz A, Schiff MH, et al. Efficacy and safety of adalimumab in patients with ankylosing spondylitis: results of a multicenter, randomized, double-blind, placebo-controlled trial. Arthritis Rheum 2006; 54: $2136-46$.

7. Inman RD, Davis JC, Jr, Heijde D, et al. Efficacy and safety of golimumab in patients with ankylosing spondylitis: results of a randomized, double-blind, placebo-controlled, phase III trial. Arthritis Rheum 2008; 58 : 3402-12.

8. Landewé R, Braun J, Deodhar A, et al. Efficacy of certolizumab pegol on signs and symptoms of axial spondyloarthritis including ankylosing spondylitis: 24-week results of a double-blind randomised placebocontrolled Phase 3 study. Ann Rheum Dis 2014; 73 (1):39-47.

9. Barkham N, Keen HI, Coates LC, et al. Clinical and imaging efficacy of infliximab in HLA-B27-Positive patients with magnetic resonance imaging-determined early sacroiliitis. Arthritis Rheum 2009; 60: 946-54.

10. Song $\mathrm{IH}$, Althoff $\mathrm{CE}$, Haibel $\mathrm{H}$, et al. Frequency and duration of drug-free remission after 1 year of treatment with etanercept versus sulfasalazine in early axial spondyloarthritis: 2 year data of the ESTHER trial. Ann Rheum Dis 2012; 71: 1212-5. 
11. Sieper J, van der Heijde D, Dougados M, et al. Efficacy and safety of adalimumab in patients with nonradiographic axial spondyloarthritis: results of a randomised placebo-controlled trial (ABILITY-1) Ann Rheum Dis 2013; 72: 815-22.

12. Dougados M, van der Heijde D, Sieper J, Braun J, et al. The symptomatic efficacy of etanercept and its effects on objective signs of inflammation in early nonradiographic axial spondyloarthritis: a multicenter, randomized, double-blind, placebo-controlled trial. Arthritis Rheumatol 2014 Aug; 66 (8): 2091-102.

13. Spadaro A, Lubrano E, Marchesoni A, et al. Remission in ankylosing spondylitis treated with anti-TNF-a drugs: a national multicentre study. Rheumatology (Oxford) 2013 Oct; 52 (10): 1914-9.

14. Haibel H, Heldmann F, Braun J, Listing J, Kupper H, Sieper J. Long-term efficacy of adalimumab after drug withdrawal and retreatment in patients with active non-radiographically evident axial spondyloarthritis who experience a flare. Arthritis Rheum 201365 (8): 2211-3.

15. Cantini F, Niccoli L, Cassarà E, Kaloudi O, Nannini C. Duration of remission after halving of the etanercept dose in patients with ankylosing spondylitis: a randomized, prospective, long-term, follow-up study. Biologics 2013; 7: 1-6.

16. Landewé R, Sieper J, Mease $P$, et al. Efficacy and safety of continuing versus withdrawing adalimumab therapy in maintaining remission in patients with non-radiographic axial spondyloarthritis (ABILITY-3): a multicentre, randomised, double-blind study. Lancet 2018 Jul 14; 392 (10142): 134-44.

17. Moreno M, Gratacós J, Torrente-Segarra V, et al. REMINEA study Group. Withdrawal of infliximab therapy in ankylosing spondylitis in persistent clinical remission, results from the REMINEA study. Arthritis Res Ther 2019 Apr 5; 21 (1):88.

18. Kaltsonoudis E, Pelechas E, Voulgari PV, Drosos AA. Maintained Clinical Remission in Ankylosing Spondylitis Patients Switched from Reference Infliximab to Its Biosimilar: An 18-Month Comparative OpenLabel Study. J Clin Med 2019 Jul 2; 8 (7). pii: E956. doi: 10.3390/jcm8070956.

19. Gratacós J, Pontes C, Juanola X, et al. REDES-TNF investigators. Non-inferiority of dose reduction versus standard dosing of TNF-inhibitors in axial spondyloarthritis. Arthritis Res Ther 2019 Jan 8; 21 (1):11. doi: 10.1186/s13075-018-1772-z.

20. Carron P, Varkas G, Renson T, Colman R, Elewaut D, Van den Bosch F. High Rate of Drug-Free Remission After Induction Therapy With Golimumab in Early Peripheral Spondyloarthritis. Arthritis Rheumatol 2018 Nov; 70 (11): 1769-77.

21. Sieper $J$, Landewé $R$, Magrey $M$, et al. Predictors of remission in patients with non-radiographic axial spondyloarthritis receiving open-label adalimumab in the ABILITY-3 study. RMD Open 2019 Jun 7; 5 (1): e000917.

22. Wanders A, Heijde Dv, Landewé R, et al. Nonsteroidal antiinflammatory drugs reduce radiographic progression in patients with ankylosing spondylitis: a randomized clinical trial. Arthritis Rheum 2005 Jun; 52 (6): $1756-65$.

23. Kroon F, Landewé R, Dougados M, van der Heijde D. Continuous NSAID use reverts the effects of inflammation on radiographic progression in patients with ankylosing spondylitis. Ann Rheum Dis 2012 Oct; 71 (10): 1623-9.

24. Sieper J, Listing J, Poddubnyy D, et al. Effect of continuous versus on-demand treatment of ankylosing spondylitis with diclofenac over 2 years on radiographic progression of the spine: results from a randomised multicentre trial (ENRADAS). Ann Rheum Dis 2016 Aug; 75 (8): 1438-43.

25. Bakland G, Gran JT, Nossent JC. Increased mortality in ankylosing spondylitis is related to disease activity. Ann Rheum Dis 2011 Nov; 70 (11): 1921-5.

26. Haroon NN, Paterson JM, Li P, Inman RD, Haroon N. Patients With Ankylosing Spondylitis Have Increased Cardiovascular and Cerebrovascular Mortality: A Population-Based Study. Ann Intern Med 2015 Sep 15; 163 (6): 409-16.

27. Ward MM, Deodhar A, Gensler LS, et al. 2019 Update of the American College of Rheumatology/Spondylitis Association of America/Spondyloarthritis Research and Treatment Network Recommendations for the Treatment of Ankylosing Spondylitis and Nonradiographic Axial Spondyloarthritis. Arthritis Care Res (Hoboken) 2019 Oct; 71 (10): 1285-99. 